\title{
Infected Mandibular Fracture; Can the Tooth Buds Be Saved?
}

\author{
Mamta Agrawal and T.S Khan \\ Department of OMFS Purvanchal Institute of Dental Sciences, GIDA \\ Gorakhpur, Uttar Pradesh, India
}

Correspondence should be addressed to: Mamta Agrawal; mamtaag02@yahoo.co.in

Received date: 13 November 2013; Accepted date: 12 January 2014; Published date: 29 May 2015

Academic Editor: Takashi Nakamoto

Copyright (C) 2015. Mamta Agrawal and T.S Khan. Distributed under Creative Commons CC-BY 4.0

\begin{abstract}
Treatment of mandibular fractures requires a different approach in children than in adults. The growth potential of jaw bones and the presence of tooth buds are the main concerns during treatment. Management of such cases is mostly by closed reduction, and long term monitoring is also required. Complications as infections and non-union are rare in children owing to the greater osteogenic potential. This paper presents a rare case of a child reporting with infection in the fracture region after trauma. Two developing teeth were present in the fracture line region. Although earlier studies advocate the extraction of tooth buds in cases of infection; (as the presence of a tooth bud may compromise the union of fractured segments) the following case was managed more conservatively. Extraction of a deciduous tooth lying in proximity of tooth buds was done, and simultaneously adequate antibiotic therapy was provided, which resulted in the resolution of the infection. Long term follow up showed proper development of the tooth buds in spite of earlier infection.
\end{abstract}

Keywords: Mandible fracture, Infection at fracture site, Tooth Bud in fracture line

\section{Introduction}

Mandibular fracture in children usually results in the involvement of a tooth bud directly in the fracture line. A study by Koenig et al (1994) proves that the injury has the potential to damage the developing tooth germ. The presence of infection may also adversely affect the developing tooth and the fracture union. Specific treatment guidelines for developing tooth buds located in the line of mandibular fractures have been suggested by Suei Y et al (2006), concluding that except in cases of infection the tooth buds should be preserved.

The management of mandibular body fractures in children depends on the fracture type and the stage of skeletal and dental development. Different treatment modalities 
include conservative non-invasive management, closed reduction with immobilization, and open reduction with internal fixation as discussed in detail by Aizenbud D et al (2009). Closed reduction is the preferred mode of treatment, and open reduction is advised only when the fracture cannot be managed closed.

At our centre, we came across a case of paediatric mandibular fracture with two developing teeth at the fracture site, resulting in infection with extra oral discharging sinus. Instead of removing the developing teeth from the fracture line, we tried an alternative treatment by extracting the deciduous tooth in vicinity which resulted in the resolution of the infection. Through this case presentation, we aim to suggest that even in cases of infected fracture, the tooth buds present in fracture line may be saved if the infection can be controlled otherwise.

\section{Case Report}

A two year old child with a history of mandibular fracture treated elsewhere by closed reduction reported at our centre 45 days after trauma. The chief complaint was of an extra oral discharging sinus since last 15 days which was not responding to medical treatment. (Figure 1)

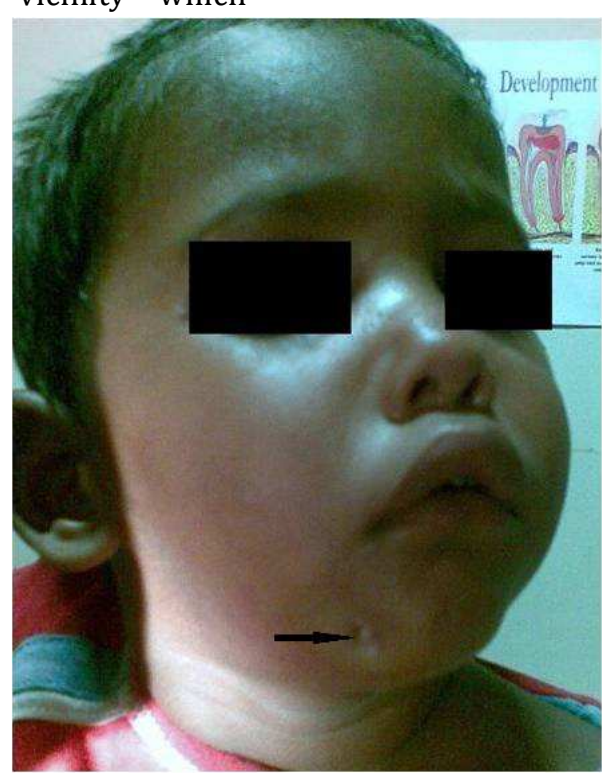

Figure 1: Child with extra oral sinus

The sinus was in the right para symphysis region near the lower border of mandible. The fracture appeared to have healed clinically as there was no apparent mobility of the fragments, and the child was able to chew properly. Radiographically; the fracture line appeared to be involving the follicles of

lateral incisors and canine (with the crowns still in developing stage). The roots of the deciduous first molar also appeared in approximation of the fracture line. (Figure 2) Although the fracture appeared to be united clinically, after radiographic interpretation the bone healing appeared compromised. (Osteogenic changes denoting union are clearly visible after the fifth week of mandibular fracture in young patients; which was not observed in this case.) 


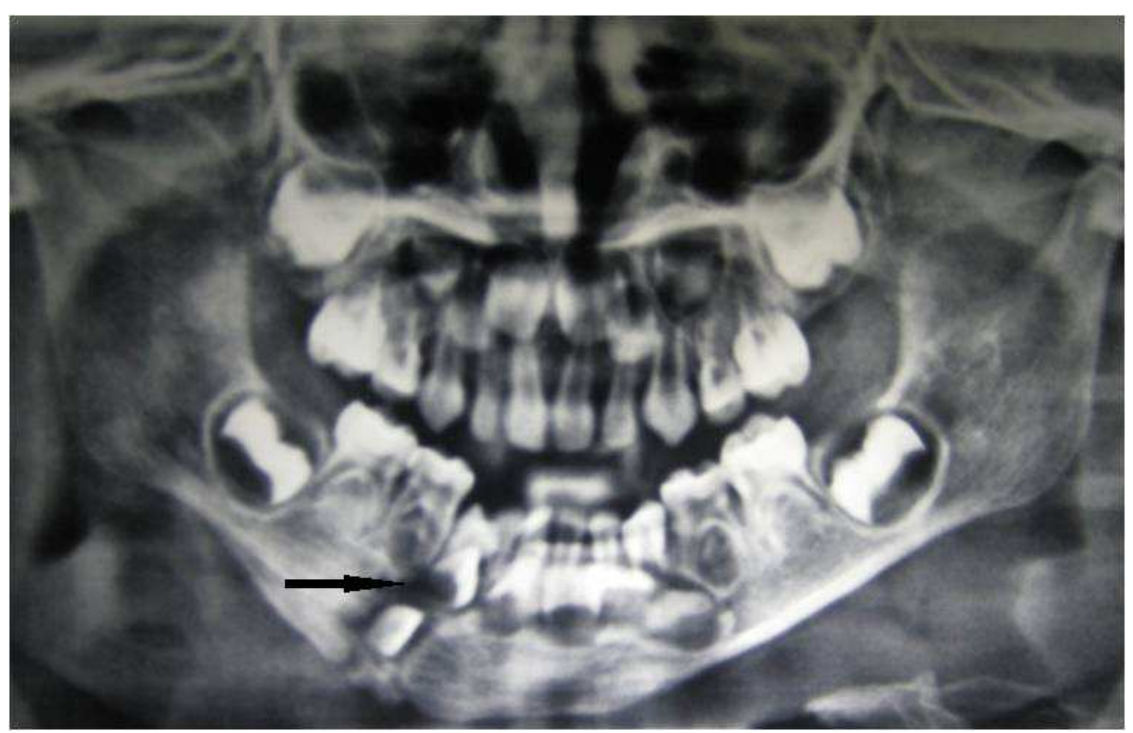

Figure 2: Pre extraction radiograph

Osteogenesis and union was the best radiographic criterion for evaluating followup radiographs. This change started to predominate 1-2 months after injury in patients less than 18 years of age (21/31, $68 \%$ ), and 2-3 months after injury in older patients $(21 / 25,84 \%)$. Overall, union was noted in 98 of the 115 patients (85\%) 3 months or more after the fracture. We recommend radiographic follow-up examination to confirm clinical judgement during the fifth week after a mandibular fracture in patients less than 18 years of age, and the ninth week for older patients. The fixation materials should be removed during the fifth month after injury.

After pus culture and sensitivity, the patient was put on antibiotics [amoxicillin 200mg and metronidazole $100 \mathrm{mg}$ three times daily for a period of 15 days]. Extraction of the deciduous molar was initially planned to provide an alternative route for extrusion of infective exudate from the bone; so as to avoid extracting the teeth present in the fracture line. Secondary surgery with open reduction and extraction of all teeth present in fracture line was decided in case of nonhealing of sinus and failure of initial treatment plan.

However the extra oral sinus healed completely and the patient was asymptomatic after extraction of deciduous molar and 15 days of antibiotic therapy. Follow up study after three months and one year showed the buds to be developing properly. (Figure 3) Long term follow up could not be done due to patient noncompliance. 


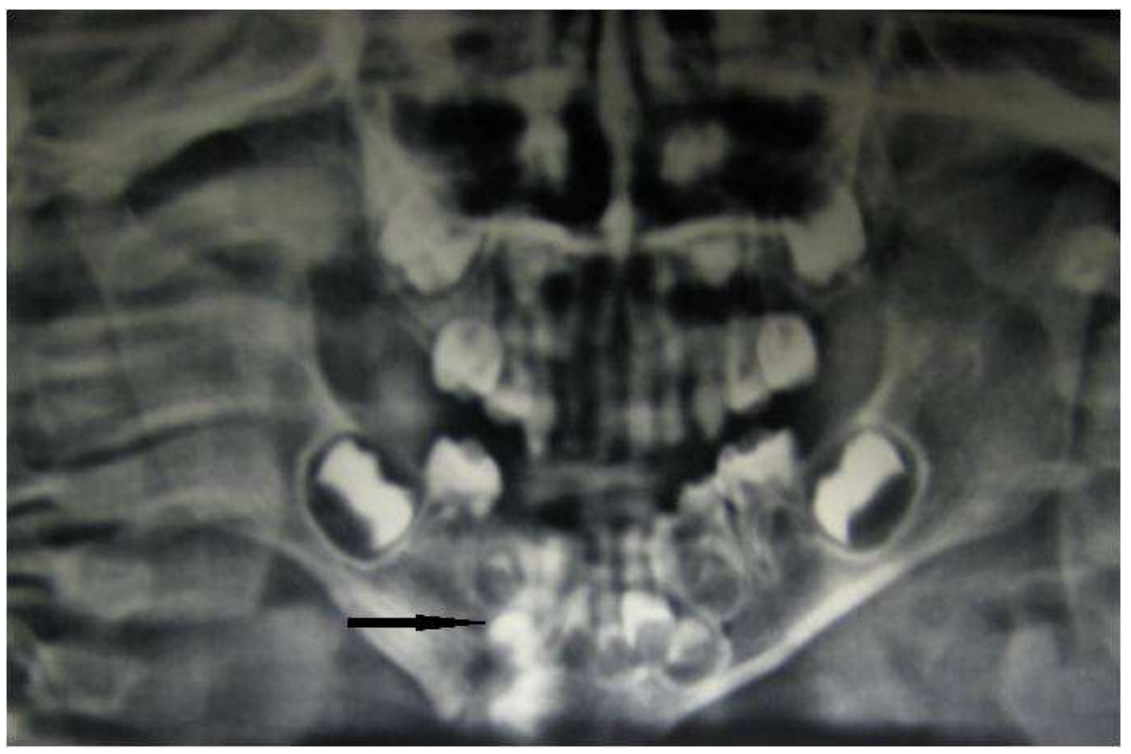

Figure 3: Radiograph after 3 months

\section{Discussion}

The incidence of abnormalities in teeth present in fracture line after dental and maxillofacial traumas is relatively high ranging from discolorations, enamel hypoplasia, crown dilacerations, disturbance in eruption and arrested root formation. Antibiotic prophylaxis is extremely important as it assists in sustaining the development of involved tooth bud and prevents infection in the fracture line.

Although infection at fracture site is very rare in children, it may be attributed to improper immobilization or inappropriate antibiotic therapy. Poor patient compliance, metabolic disturbances, generalized disease related to inadequate immobilization of fracture segments, and inadequate reduction of the fracture segments may also result in infection.

In our case, the patient had a discharging extra oral sinus indicating infection at the fracture site. The infection did not seem to be arising from the first deciduous molar as the tooth did not have any lesion and the radiograph was suggestive of compromised bone healing along the entire mandibular bony height. Here, our main concern was control of infection and proper fracture union. Two tooth buds were present in the fracture line, and the deciduous molar root was also in the same vicinity, so it was presumed that the extraction of that tooth may form a portal for escape of infective exudates, and thereby assist in resolving the infection. Proper antibiotic prophylaxis was given before and after extraction, and after a period of 15 days the patient was totally asymptomatic. Follow up radiographs showed proper union of segments and continued development of tooth buds indicating this to be a feasible option for saving permanent tooth buds in cases of infection.

The development of tooth buds in fracture line cannot be predicted. We suggest it may be prudent to try and save the bud. A single case study is not sufficient to reach to any conclusion; additional studies are required for evidence based management protocol in such cases. 


\section{References}

1- Aizenbud D, Hazan-Molina H, Emodi O and Rachmiel A. (2009) "The management of mandibular body fractures in young children," Dental Traumatol, 25: 565-70

2- Koenig W.R, Olsson A.B. and Pensler J.M. (1994) "The fate of developing teeth in facial trauma: Tooth buds in the line of mandibular fractures in children." Ann Plast Surg 32:503505

3- Suei Y, Mallick P.C, Nagasaki T, Taguchi A, Fujita M and Tanimoto K. (2006) "Radiographic evaluation of the fate of developing tooth buds on the fracture line of mandibular fractures." J Oral Maxillofac Surg, 64: 94-99. 